\title{
Bilateral papillitis and unilateral focal chorioretinitis as the presenting features of syphilis
}

\author{
Christy Elizabeth Benson ${ }^{1}$, Mohamed Kamel Soliman ${ }^{1,2}$, Alexander Knezevic ${ }^{1}$, Daisy Ding Xü ${ }^{3}$, Quan Dong Nguyen ${ }^{1}$ \\ and Diana $\vee \mathrm{Do}^{1 *}$
}

\begin{abstract}
Background: Syphilis is a multisystem bacterial infection caused by Treponema pallidum. The incidence of infection in the United States has risen by more than 75\% since the year 2000, when it was at a low of 2.1 per 100,000 people. Ocular involvement may occur in any stage of infection and may present in a variety of ways, with posterior uveitis being the most common manifestation. We report a case of ocular syphilis infection with an unusual presentation of bilateral non-granulomatous panuveitis with papillitis and unilateral focal chorioretinitis.
\end{abstract}

Findings: This is a retrospective case report with literature review. A 39-year-old Caucasian female presented with a 2-week history of bilateral ocular flashes and left eye pain. Dilated fundus examination revealed mild optic disc edema in both eyes, the right eye more than the left. In the left eye, there was an area of retinal elevation and whitening involving the peripheral retina. Fluorescein angiography, B-scan ultrasonography, and ocular coherence tomography were performed, and laboratory tests were ordered based on the clinical presentation. After rapid plasma reagin (RPR) and fluorescent treponemal antibody absorption (FTA-Abs) were positive, syphilitic uveitis was confirmed, and the patient was admitted for a 14-day course of high-dose intravenous penicillin G.

Conclusions: The first signs and symptoms of syphilis may be ocular, which can lead to a diagnostic challenge. A high index of suspicion is the key for early diagnosis of ocular syphilis. Prompt treatment with intravenous penicillin $\mathrm{G}$ is highly effective in resolving the infection.

Keywords: Syphilis; Retinitis; Chorioretinitis; Uveitis; Panuveitis; Papillitis

\section{Findings}

Syphilis is a multisystem bacterial infection caused by the spirochete Treponema pallidum [1]. It is primarily a sexually transmitted disease; however, contacts with an infected lesion and blood transmission are also potential routes of infection. The classic clinical course of acquired syphilis is divided into four stages: primary, secondary, latent, and tertiary syphilis [2]. The eye can be affected in any stage of infection and virtually all ocular tissues can be affected.

Uveitis occurs in approximately $10 \%$ of cases of secondary syphilis and in up to $5 \%$ of cases who have progressed

\footnotetext{
* Correspondence: diana.do@unmc.edu

${ }^{1}$ Stanley M Truhlsen Eye Institute, University of Nebraska Medical Center, 3902 Leavenworth Street, Omaha, NE 68105, USA

Full list of author information is available at the end of the article
}

to tertiary syphilis $[3,4]$. The uveitis that occurs with syphilis may be granulomatous or non-granulomatous [5], and it can affect one or both eyes in the anterior, intermediate, or posterior segments.

Syphilis earns its name as the 'great masquerader' in its ability to produce a myriad of signs and symptoms that may mimic various diseases [6]; therefore, syphilis should be kept in the differential diagnosis of ocular inflammation. Unfortunately, when ophthalmologic involvement becomes the presenting signs and symptoms, the proper diagnosis and treatment may be delayed [3,7]. Such a delay in treatment may result in irreversible visual loss and significant systemic morbidity.

\section{空}

C 2015 Benson et al.; licensee Springer. This is an Open Access article distributed under the terms of the Creative Commons Attribution License (http://creativecommons.org/licenses/by/4.0), which permits unrestricted use, distribution, and reproduction in any medium, provided the original work is properly credited. 


\section{Case report}

A 39-year-old healthy Caucasian female from rural Nebraska presented with a 2-week history of bilateral flashes and left eye pain. This was associated with redness in both eyes. The patient was generally healthy with no history of eye discharge or trauma. An extensive review of symptoms was performed, which was negative. There was no previous history of eye diseases or surgery. The patient had no chronic medical conditions.

On examination, the patient's visual acuity was 20/20 in both eyes. Slit-lamp examination revealed $1+$ conjunctival injection in the right eye and $2+$ in the left eye. The corneas were clear in both eyes. Anterior chamber examination using slit-lamp biomicroscopy showed $0.5+$ and $2+$ cells (non-granulomatous) and flare in the right and left eyes, respectively. The pupils were equally round and reactive to light without evidence of relative afferent papillary defect in either eye. There was trace nuclear sclerosis and pigment deposits in the crystalline lens of the left eye and $1+$ vitreous cell in the left eye. Intraocular pressure was within normal limits in both eyes. Fundus examination revealed mild optic disc edema in both eyes, the right eye more than the left, with 0.3 cup-todisc ratio of both eyes. There was a subtle retinal elevation and well-defined area of whitening involving the retina in the left eye. The macula and vessels showed no visible abnormalities in either eye (Figure 1A,B).

Fluorescein angiography showed hyperfluorescence corresponding to the peripheral whitening with perivascular leakage in the left eye and optic disc leakage in both eyes (Figure 2A,B,C). B-scan of the superonasal quadrant did not reveal a corresponding elevation or abnormality (Figure 3A,B). OCT of the lesion revealed retinal infiltration with hyperreflective dots (Figure 4).

Anterior chamber paracentesis was performed at the slit lamp and sent for herpes simplex virus (HSV) and varicella zoster virus (VZV) PCR and gram stain. Other lab tests were ordered including complete blood count, complete metabolic panel, erythrocyte sedimentation rate, C-reactive protein, syphilis serology, HSV and VZV titer, human immunodeficiency virus (HIV) antibodies, chest X-ray, and MRI of the brain and orbit with and without contrast. The patient was not treated with steroids while waiting for the laboratory results to return.

Acute-phase reactant was elevated, HIV antibodies were negative, and rapid plasma regain (RPR) and fluorescent treponemal antibody absorption (FTA-Abs) were both reactive. After the diagnosis was confirmed to be syphilitic uveitis, the patient was admitted for high-dose intravenous penicillin G 24 million units per day. Lumbar puncture was recommended to evaluate for CSF antibodies, but the patient declined this invasive test. After the complete 2-week course of parenteral therapy, her ocular findings resolved dramatically.

\section{Discussion}

Uveitis is the most common ocular manifestation of syphilis and is bilateral in more than $50 \%$ of cases; however, syphilitic uveitis is considered a rare cause of uveitis, accounting for $1.6 \%$ to $4.5 \%$ of cases [8].

The most common presentation of syphilitic uveitis varies between several reports $[5,9,10]$. According to a review article of 143 patients with syphilitic uveitis, the most common presentation is posterior uveitis followed by panuveitis [11]. Panuveitis, as seen in our case, most commonly occurs during the second stage of syphilis. Although the presentation may vary greatly between affected patients, there are certain features considered to be characteristic of syphilitic uveitis. As in our case, ground glass retinal opacification associated with retinal vasculitis is considered to be characteristic for syphilitic uveitis. Another distinctive feature described is acute syphilitic posterior placoid chorioretinitis (ASPPC) [12].

Standard testing used to screen for syphilis include nontreponemal tests of Venereal Disease Research Laboratory (VDRL) and RPR labs; however, these tests are nonspecific and may yield false positive results due to cross-reactivity. The gold standard tests used to confirm infection include FTA-Abs and dark field microscopy of the tissue [3]. Additionally, syphilis increases the risk of HIV transmission by
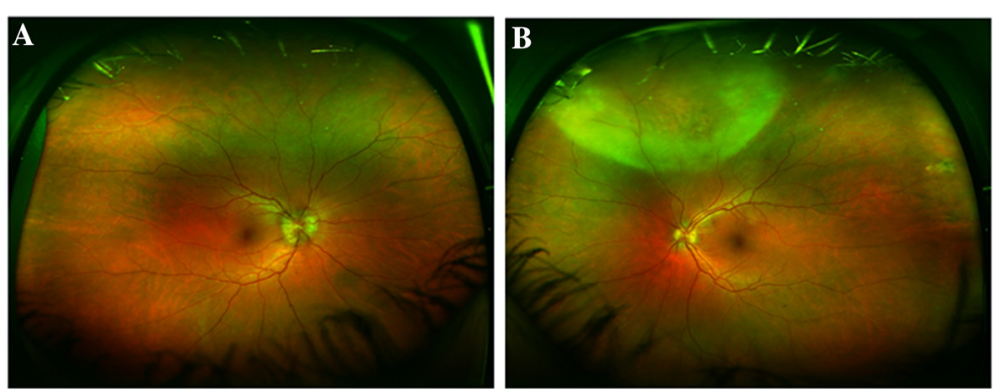

Figure 1 Fundus photograph of the right and left eyes. (A) Wide field color fundus photograph of the right eye: blurred optic disc margin. (B) Wide field color fundus photograph of the left eye: well-defined area of whitening involving the peripheral superonasal quadrant with slight haziness extending from that area up to the upper margin of disc and upper temporal arcade. The disc margin is ill-defined. 

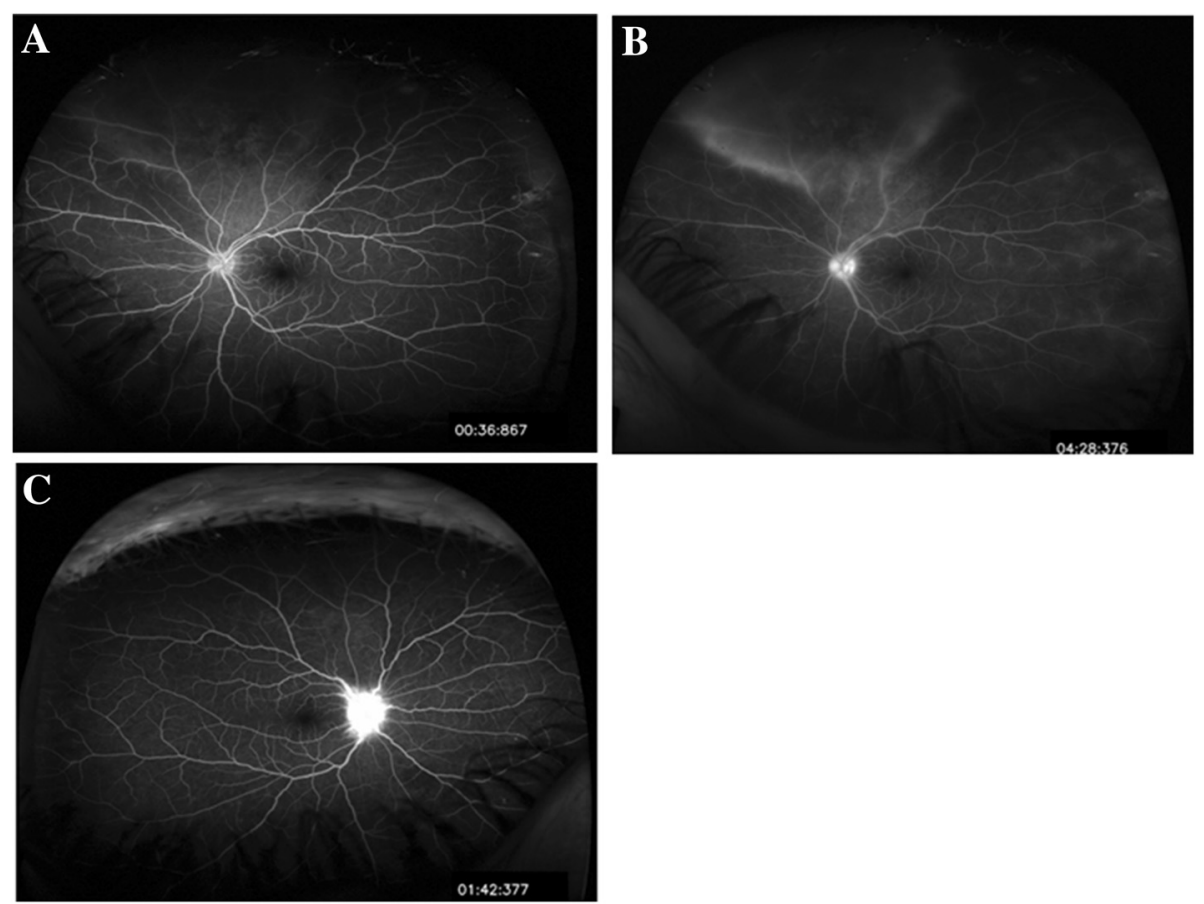

Figure 2 Fluorescein angiography of the left and right eyes. (A) Fluorescein angiography of the left eye: early hyperfluorescence corresponding to the area of retinal whitening and haziness. (B) Fluorescein angiography of the left eye: late frame shows perivascular leakage in the same area (vasculitis) together with late hyperfluorescence of the disc. (C) Fluorescein angiography of the right eye: late hyperfluorescence and leakage from the disc.

two to five times, and co-infection is common; therefore, every patient diagnosed with syphilis should also be tested for HIV [11].

Since the optic nerve and retina are considered to be extensions of the CNS, ocular syphilis is regarded as a variant of neurosyphilis; thus, every patient with syphilitic uveitis should undergo lumbar puncture and CSF analysis for the detection of neurological involvement [13]. However, some authors argue that this is only necessary with neurological symptoms or higher RPR titre values [14]. Cerebrospinal fluid findings indicative of tertiary syphilis include greater than five white blood cells per microliter, elevated CSF protein levels, and treponemal or nontreponemal antibodies [15].

According to the CDC, Nebraska is ranked 48 among 50 states reporting at least one case of primary and secondary syphilis with 0.4 cases per 100,000 populations compared to the U.S. rate of 5.0. The rate among males was 0.8 cases per 100, 000 population compared to the U.S. male rate of 9.3. The rate among females was 0.1 compared to the U.S. female rate of 0.9 which show how rare syphilis is in this particular area.
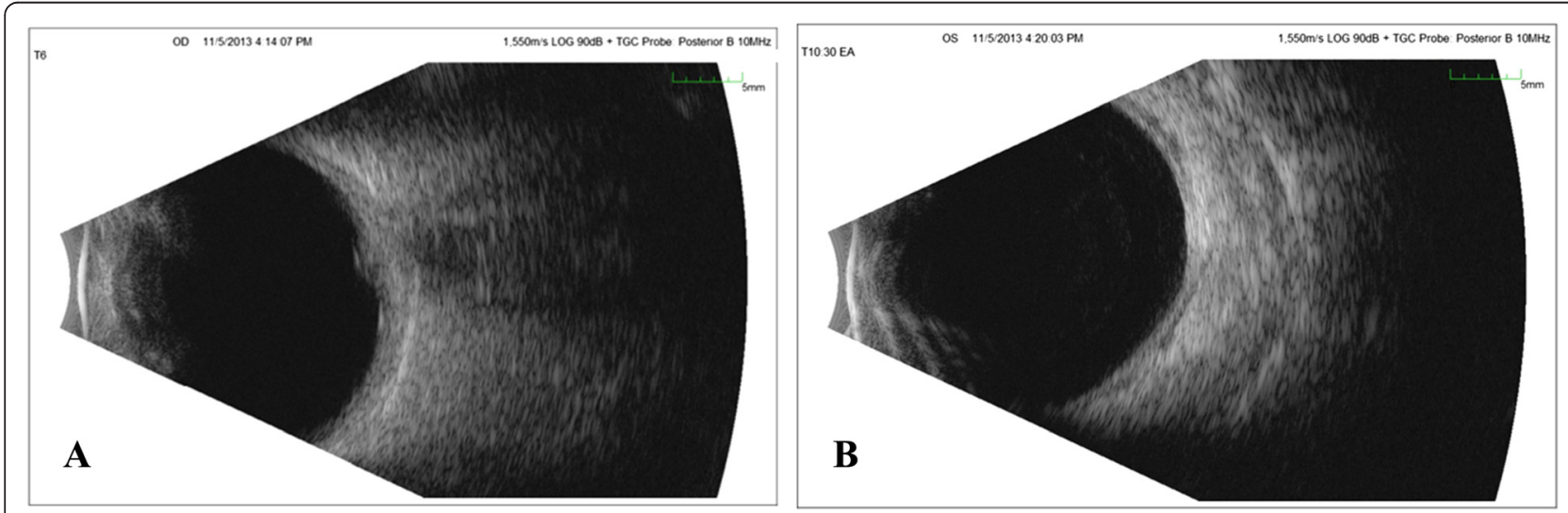

Figure 3 B-scan ultrasound of the right and left eyes. (A) B-scan ultrasound of the right eye: elevation of optic nerve head. (B) B-scan ultrasound of the left eye: no visible elevation could be appreciated in the superonasal quadrant of the left eye. 


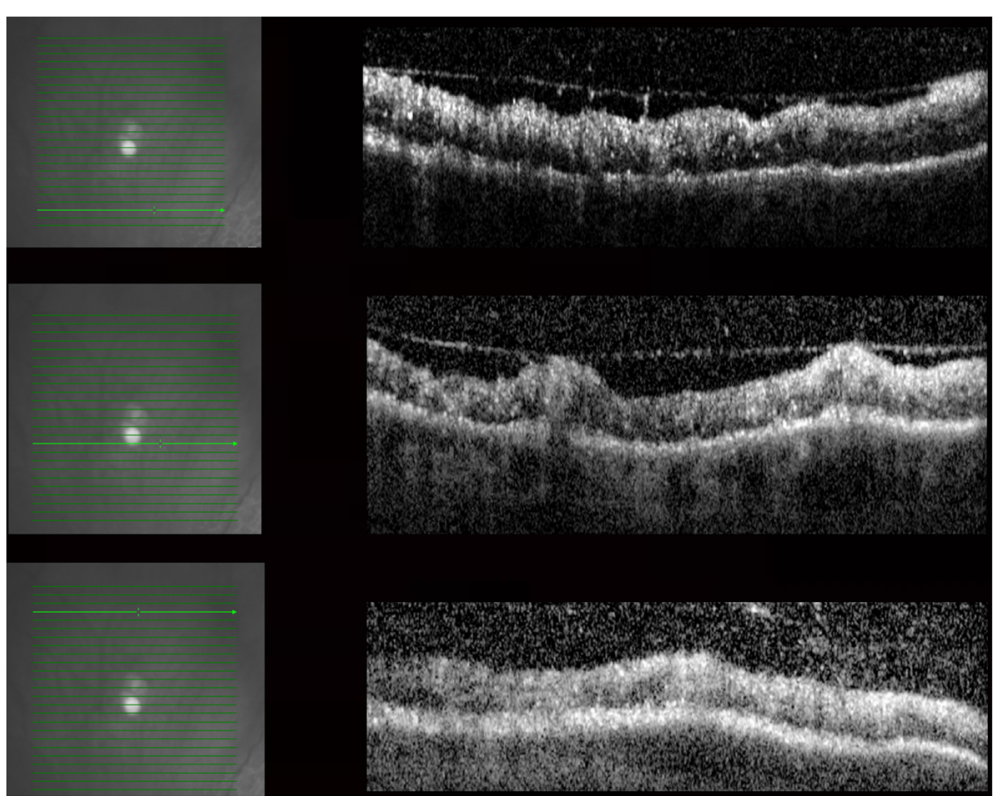

Figure 4 OCT of the lesion: irregular retinal contour with areas of retinal elevation. The individual retinal layers could not be distinguished due to infiltration with multiple hyperreflective dots. Diffuse thickening at the retinal nerve fiber layer. Irregular vitreo-retinal interface with traction by partial PVD together with moderate hyperreflective dots in the vitreous.

The CDC recommends high-dose IV penicillin G 18 to 24 million units per day for 10 to 14 days. For HIV-positive patients, they also recommend an additional treatment of intramuscular benzathine penicillin at a dose of 2.4 million units weekly for 3 weeks. If severe penicillin allergic, one can consider ceftriaxone, oral doxycycline, or azithromycin. The Jarisch-Herxheimer reaction (JHR) can occur in up to a third of neurosyphilis patients following penicillin therapy [16]. The reaction usually includes fever, sweating, and temporary worsening of symptoms of disease. Some authors suggest the use of steroids prior to antibiotics in cases of severe neurosyphilis to prevent JHR [16].

Despite the rarity of the disease in certain areas, syphilis serology should be routinely done in every case of uveitis that requires investigation. Intravenous penicillin $\mathrm{G}$ is a highly effective treatment resulting in a dramatic improvement [17]; thus, early diagnosis and prompt treatment of syphilitic uveitis prevents potential irreversible complications.

\section{Consent}

Written informed consent was obtained from the patient.

\section{Abbreviations}

CDC: Centers for Disease Control and Prevention; OCT: optical coherence tomography; PCR: polymerase chain reaction.

\section{Competing interests}

The authors declare that they have no competing interests.

\section{Authors' contributions}

CEB: Acquisition, analysis and interpretation of data, and drafting the manuscript. MKAS conceived and designed the study; acquired, analyzed, and interpreted the data; and drafted the manuscript. AK wrote the abstract and participated in drafting the manuscript. DDX acquired the data. QDN revised the manuscript. DVD acquired the data, revised the manuscript, and gave final approval of the version to be published. All authors read and approved the final manuscript.

\section{Disclosure}

This paper is presented as a case report at the FIOS meeting, ARVO on May 2014 at Orlando, FL, USA.

\section{Author details}

'Stanley M Truhlsen Eye Institute, University of Nebraska Medical Center, 3902 Leavenworth Street, Omaha, NE 68105, USA. ${ }^{2}$ Department of Ophthalmology, Assiut University Hospital, Al Gamaa St, Assiut 71516, Egypt. ${ }^{3}$ Tenth People's Hospital, 301 Yanchang Road, Shanghai District, Shanghai 200072, China.

Received: 15 August 2014 Accepted: 30 March 2015

Published online: 05 June 2015

\section{References}

1. Margo CE, Hamed LM (1992) Ocular syphilis. Surv Ophthalmol 37(3):203-220

2. Deschenes J, Seamone CD, Baines MG (1992) Acquired ocular syphilis: diagnosis and treatment. Ann Ophthalmol 24(4):134-138

3. Aldave AJ, King JA, Cunningham ET Jr (2001) Ocular syphilis. Curr Opin Ophthalmol 12(6):433-441

4. Jumper JM, Machemer R, Gallemore RP, Jaffe GJ (2000) Exudative retinal detachment and retinitis associated with acquired syphilitic uveitis. Retina (Philadelphia, Pa) 20(2):190-194

5. Barile GR, Flynn TE (1997) Syphilis exposure in patients with uveitis. Ophthalmology 104(10):1605-1609

6. Meehan K, Rodman J (2010) Ocular perineuritis secondary to neurosyphilis. Optom Vis Sci 87(10):E790-E796, Doi:10.1097/OPX.0b013e3181f361b0

7. Kiss S, Damico FM, Young LH (2005) Ocular manifestations and treatment of syphilis. Semin Ophthalmol 20(3):161-167, doi:10.1080/08820530500232092

8. Tait IA (1983) Uveitis due to secondary syphilis. Br J Vener Dis 59(6):397-401

9. Thomas S, Wiselka M, Dhar J, Bibby K (2006) Syphilis presenting as acute multifocal retino-choroiditis. J R Soc Med 99(7):371-372, doi:10.1258/jrsm.99.7.371 
10. Deschenes J, Seamone C, Baines M (1990) The ocular manifestations of sexually transmitted diseases. Can J Ophthalmol J Canadien d'ophtalmologie 25(4):177-185

11. Amaratunge BC, Camuglia JE, Hall AJ (2010) Syphilitic uveitis: a review of clinical manifestations and treatment outcomes of syphilitic uveitis in human immunodeficiency virus-positive and negative patients. Clin Exp Ophthalmol 38(1):68-74, doi:10.1111/j.1442-9071.2010.02203.x

12. Gass JD, Braunstein RA, Chenoweth RG (1990) Acute syphilitic posterior placoid chorioretinitis. Ophthalmology 97(10):1288-1297

13. Browning DJ (2000) Posterior segment manifestations of active ocular syphilis, their response to a neurosyphilis regimen of penicillin therapy, and the influence of human immunodeficiency virus status on response. Ophthalmology 107(11):2015-2023

14. Libois A, De Wit S, Poll B, Garcia F, Florence E, Del Rio A, Sanchez P, Negredo E, Vandenbruaene M, Gatell JM, Clumeck N (2007) HIV and syphilis: when to perform a lumbar puncture. Sex Transm Dis 34(3):141-144, doi:10.1097/01.olq.0000230481.28936.e5

15. Jay CA (2006) Treatment of neurosyphilis. Curr Treat Options Neurol 8(3):185-192

16. Kojan S, Van Ness PC, Diaz-Arrastia R (2000) Nonconvulsive status epilepticus resulting from Jarisch-Herxheimer reaction in a patient with neurosyphilis. Clin Electroencephalogr 31(3):138-140

17. Workowski KA, Berman SM (2006) Sexually transmitted diseases treatment guidelines, 2006. MMWR Recommendations and reports: morbidity and mortality weekly report. Recommendations and reports/Centers for Disease Control 55 (RR-11). pp 1-94

\section{Submit your manuscript to a SpringerOpen ${ }^{\circ}$} journal and benefit from:

- Convenient online submission

- Rigorous peer review

- Immediate publication on acceptance

- Open access: articles freely available online

- High visibility within the field

- Retaining the copyright to your article 